\title{
Individual differences in sleep patterns
}

\author{
D. R. DAVIES \\ B.A., Ph.D., F.B.Ps.S. \\ Department of Applied Psychology, University of Aston in Birmingham
}

\begin{abstract}
As Johnson (1973) has remarked, the ultimate reason for studying sleep is to gain a more comprehensive understanding of waking behaviour. The assumption underlying this observation is that sleeping and waking behaviours are related and there are at least three main ways in which the relationship of sleep parameters to subsequent waking behaviour can be established. Firstly, individual differences in sleep parameters can be linked to individual differences in subjective reports about sleep, for example, to subjective reports of sleep quality; secondly, the attempt can be made to relate individual differences in sleep parameters to individual characteristics such as age, sex, personality and intelligence, which are known to be related to individual differences in waking behaviour; thirdly, individual differences in sleep parameters can be linked to individual differences in subsequent performance on a variety of tasks requiring different skills. This paper sets out to provide a brief and selective review of individual differences in sleep patterns. It is divided into four parts. The first examines ways in which patterns of sleep have been defined and the methods of studying them that have typically been employed. The second and third concentrate on the relation between patterns of sleep and reports of sleep quality and on that between sleep patterns and individual characteristics such as age, sex and personality. In conclusion, the importance of sleep patterns for waking behaviour is discussed.
\end{abstract}

\section{Sleep patterns: definition and measurement}

The more popular present-day methods employed in the investigation of sleep patterns are, firstly, the continuous recording of the electrical activity of the brain, using the electroencephalogram or EEG and secondly, the collection of information concerning the duration and distribution of sleep through the use of sleep logs or diaries. In addition to these two main methods, other methods such as continuous visual observation, interviews and questionnaires are occasionally employed. Frequently, recordings of muscle activity, eye movement activity and cardiovascular and electrodermal activity are also taken in conjunction with the EEG.

Continuous EEG recording provides the most objective and reliable information concerning the activity of brain during sleep, although as a method of collecting data about sleep it is expensive. Usually only one or two individuals can be monitored at any one time. The use of sleep logs or diaries permits data on a large number of people to be gathered, often over a period of weeks or months, but this method depends upon the individual's own report of the amount of sleep obtained at different times of day and reports of sleep quality are seldom obtained except by counting the number of mid-night awakenings. However, relatively long term differences in patterns of sleep can be related to age, sex and personality differences using this method.

The sleep diary method thus provides information on the duration and distribution of nocturnal and daytime sleep over relatively long periods. The EEG on the other hand, provides more detailed information concerning the electrical activity of the brain during nocturnal sleep. As is well known, theseo patterns of activity are not uniform and the EEG? activity of sleep has been classified into stages. Loomis, Harvey and Hobart (1937) were the first to attempt to divide sleep into stages using EEG criteria, and Dement and Kleitman (1957) later proposed a slightly modified system of sleep stage identification. Rechtschaffen and Kales (1968), in their standardization of the methodology of sleep stage identification, proposed some revisions and refinements of the system employed by Dement and Kleitman. Six sleep stages are generally delineated and, to supplement their EEG identification, the electro-oculogram (EOG) and submental electromyogram (EMG) are utilized. These stages are Stage W (waking) Stages 1-4, and Stage 1-REM (rapid eye movement sleep). Normally the all-night EEG record is divided up into $1 \mathrm{~min}$ epochs for scoring purposes and each epoch is categorized as containing one of the six sleep stages. However, there are many difficulties associated with the scoring of sleep stages and the utility of the concept has sometimes been questioned. Considerable variability is found in the scoring of Stage 3 and a number of different criteria appear to have been used. Frequently, Stages 3 and 4 are scored together as one category of 'delta' or 'slow-wave' sleep (SWS). It is generally assumed that sleep stages vary in depth, the depth of sleep becoming progressively greater from Stage 1 to Stage 4. 
Again, there is both agreement and disagreement concerning the estimates of sleep depth provided by different methods, particularly with respect to REM sleep.

In young adults approximately $50 \%$ of the total sleep time is spent in Stage 2, about $20 \%$ in Stages 3 and 4 (Delta sleep), 25\% in REM sleep and the remaining $5 \%$ or so in Stages 0 and 1 . Delta sleep and REM sleep are also differentially distributed within nocturnal sleep. The former occurs predominantly in the first half of the sleep period and successive delta sleep periods become progressively shorter. REM sleep on the other hand, is found predominantly, although not exclusively, in the second half of the night and successive REM periods, which follow a roughly 90 -min cycle, become progressively longer.

Individuals can thus be compared in terms of their EEG sleep profiles, for instance, the percentage of total sleep time spent in each sleep stage, the number of sleep stage shifts, the number of awakenings, the amount of time spent awake, sleep onset latency, latency to the first REM period, REM cycle length and so on. In addition, comparisons in terms of other physiological variables recorded during sleep can also be made. Data concerning individual differences in EEG sleep patterns can then be related to other dimensions of individual differences, to subsequent task performance, ratings of mood and to subjective reports of sleep quality.

\section{Sleep patterns and the quality and duration of sleep}

In a now classic study, Monroe (1967) investigated the sleep of self-reported 'good' and 'poor' sleepers, using a sample of men in their mid-twenties, selected from a larger population on the basis of their answers to a questionnaire. Each group ('good' and 'poor' sleepers) was composed of sixteen people. Good sleepers described themselves as usually falling asleep in less than $10 \mathrm{~min}$ and as never taking more than $15 \mathrm{~min}$; they hardly ever woke up during the night and, if they did, almost always experienced no difficulty in falling asleep once more. Poor sleepers, on the other hand, described themselves as normally taking about $60 \mathrm{~min}$ to fall asleep and as always taking more than $30 \mathrm{~min}$; they woke up on average at least once during the night and if they did so, experienced great difficulty in returning to sleep. Each member of these two groups subsequently slept in a sleep laboratory for $7 \mathrm{hr}$ on two nights.

Monroe found that the good sleepers, compared to poor sleepers spent more time asleep, spent less time in lighter sleep, particularly Stage 2, and more time in REM sleep. They awoke less often and fell asleep more rapidly. However, the proportion of time spent in delta sleep was about the same for the two groups, although poor sleepers took longer to reach it. This group also took longer to reach the first REM period of the night. The motility of poor sleepers was also higher, particularly in the second half of the night and rectal temperature was higher throughout the night. In general the level of physiological activity was higher for poor sleepers not only during sleep, but also during wakefulness.

Monroe observed that differences in the quality of sleep normally obtained by good and poor sleepers may be more marked than is suggested by his study. Most of the good sleepers rated their laboratory sleep as worse than the sleep they typically obtained. The majority of poor sleepers, on the other hand, rated it as considerably better.

This study makes clear that differences between self-reported good and poor sleepers are not merely at the level of verbal report, but that there can be marked differences in their sleep profiles. Nevertheless, some evidence suggests that assessments of sleep quality depend more on the subjective recall of a night's sleep and less on objective measures recorded during sleep (Hauri, 1970)-a view that accords well with common sense.

Differences have also been observed in a number of studies between self-reported 'long' and 'short' sleepers (Hartmann, 1973). The former are defined as individuals who report themselves as normally sleeping for more than $9 \mathrm{hr} / \mathrm{night}$ and the latter as sleeping for less than $6 \mathrm{hr}$, these reports being checked against sleep diaries. Comparisons of the sleep profiles of these two groups with those of 'normal' sleepers have shown that differences between long, short and normal sleepers tend to be principally in the amount of REM sleep taken, long sleepers having more REM sleep than the other two groups. Compared to normals, long sleepers tend to spend more time awake and in REM sleep, while short sleepers spend less time in Stages 1, 2, 3 and REM sleep. The REM periods of long sleepers also appear to be more 'intense', as inferred from REM density measures and personality tests suggest that this group is more anxious and introverted, while short sleepers tend to be more extroverted and sociable. Hartmann suggested, in fact, that his 'long sleepers' could be equated with Monroe's 'poor sleepers'.

\section{Age, sex and sleep profiles}

A number of studies, employing both EEG sleep profiles and sleep diaries, have examined age and sex differences in patterns of sleep. One of the most thorough reports is that of Williams, Karacan and Hursch (1974), which provides a detailed account of series of studies conducted at the University of Florida. Sleep profiles were collected from eleven different age groups, ranging from 3- to 5-year-old children to individuals in their seventies, each group 
consisting of approximately ten men and ten women. Some of the major findings are briefly summarized below.

Among 3- to 5-year-olds total sleep time was greater for boys than for girls, as was the time spent in Stage 2 sleep. Between the ages of 6 and 19, however, few sex differences in sleep profiles emerged. In the twenties, men experienced more nocturnal awakenings than did women and also less Stage 2 sleep, and from the age of 40 onwards both total sleep time and the amount of REM sleep were greater for women than for men. Women also awoke less frequently and obtained more delta sleep. Williams et al. (1974) observed that, after the age of 30 , consistently fewer men than women were found to exhibit Stage 4 and in the oldest group they tested Stage 4 was completely absent in men, while being present in five out of ten women.

With age, total sleep time fell markedly, both within the age range 3-12 years and within the age range 12-20 years. Thereafter, from the twenties to the seventies a slight fall in total sleep time occurred, being somewhat more marked in men than in women. The time spent awake tended to increase and in terms of a 'sleep efficiency' index calculated by dividing total sleep time by time in bed, a gradual reduction with age from about the age of 30 for men and 50 for women was observed, which became more marked for both sexes from the mid-fifties onwards. The number of awakenings averaged less than two per night for both sexes from the age of 3 to the early teens. It then increased gradually for women up to the age of 70 and for men up to the age of 40 and thereafter rose sharply in both cases. For both sexes, too, the number of sleep stage shifts increased in age, and reductions in the amounts of both REM sleep and delta sleep were observed.

In general, these findings with respect to age complement other EEG studies of age differences in sleep patterns which have found reductions in both REM and particularly delta sleep to occur (Feinberg and Carlson, 1968; Feinberg, Koresko and Heller, 1967; Kahn and Fisher, 1969). However, it seems clear from the studies by Williams et al. (1974) that men appear to experience more disturbed EEG sleep at an earlier age than do women and the decline with age is more marked.

A number of studies using questionnaires and sleep diaries have also examined age and sex differences in sleep patterns. Sometimes, the results of such studies have tended to complement and extend results obtained from EEG sleep studies; sometimes conflicting results have been produced. Webb (1965), using a questionnaire, and Tune $(1968,1969)$, using the sleep diary method, both found that, in general, older individuals reported themseves as taking somewhat more sleep per $24 \mathrm{hr}$ period than did younger ones. Tune's study was based on an 8 week sleep diary, in which the sleep obtained was recorded for each 24-hr period in 30-min blocks in which subjects subsequently judged themselves to have been asleep for more than $15 \mathrm{~min}$. Data were collected from a total of 509 subjects drawn from a volunteer panel on Merseyside, although since this sample was biased, containing more men than women and the mean age was fairly high, data for a selected sample were subsequently analysed consisting of 240 subjects with twenty men and twenty women in each decade group from the twenties to the seventies. These age groups were matched as far as possible for temperament, in terms of Heron Inventory Scores (Heron, 1956) and for non-verbal intelligence. A number of significant correlations between age, sex, temperament and sleep variables were observed. Age correlated positively and significantly with the number of nocturnal awakenings and with the number and duration of daytime naps. For the sample as a whole, the frequency and length of nocturnal awakenings on the one hand and of daytime naps on the other hand were significantly and positively related, suggesting that sleep lost at night tends to be made up in the day. This is presumably easier for some age groups to achieve than others.

The data were also analysed by analysis of variance and by $\chi^{2}$. Analyses of variance were applied to the mean duration of sleep, the mean time of falling asleep and the mean time of awakening, the distributions of all these variables being normal. These analyses showed that less nocturnal sleep was obtained with age and by introverts and that these two variables interacted. Up to the age of 40 no difference was apparent in the amount of sleep reported by the two temperament groups but thereafter the amount reported by extroverts increased with age while that reported by introverts fell. A tendency both to fall asleep and to awaken earlier with age was also noted. However, no difference between temperament groups in terms of the time of falling asleep was found, while after the age of 40 introverts tended to awaken progressively earlier.

There was a large increase in the number and duration of nocturnal awakenings with age. In the twenties an average frequency of once per 2 weeks was found whereas in the seventies an average figure of over three per week was reported. Individuals who reported most nocturnal disturbance tended to be women over the age of 50 . The mean number of daytime naps also increased with age rising from less than one per 2 weeks in the twenties to over two per week in the seventies. Over $60 \%$ of the sample reported one or more daytime naps during the 8 weeks of the study. The length of nap taken also increased 
with age. Naps accounted for less than $1 \%$ of total sleep time in the twenties but for over $6 \%$ in the seventies.

Briefly summarizing Tune's results, the amount of sleep reported as being obtained per 24-hr period appears to fall quite sharply with age from the twenties to the fifties and thereafter rises, the increase largely consisting not of more nocturnal sleep but of an increased frequency and duration of daytime naps. Men appear to avail themselves of this extra sleep to a slightly greater extent than do women. Temperament also seems to be an important modifier of the effects of age on sleep patterns and both the sex and temperament effects are explained by Tune in terms of occupational and domestic demands and social factors. However, the major difference between his results and those of Williams et al., reported above, is in the relation of nocturnal sleep disturbance to sex and age. Tune's results suggest that women, particularly after the age of 50 , are much more prone than men to report sleep disturbances in the form of the frequency and duration of nocturnal awakenings. The studies of Williams et al. suggest the reverse, that men show 'ageing effects' in the form of disturbances in their sleep patterns much earlier than do women. One of their explanations is also in terms of social factorsthat the life styles of the majority of men are more 'stressful' than are those of women, although they also suggest that hormonal differences may be important influences on EEG sleep patterns, as indeed has been shown in studies of the sleep of women during pregnancy and menstruation. It would clearly be useful, although extremely difficult, given the present state of knowledge, for some synthesis to be effected between social and biological hypotheses concerning the determinants of individual differences in sleep patterns.

\section{Conclusions}

By way of conclusions, the implications of individual differences in sleep patterns for waking behaviour will be briefly considered. One assumption that could be made is in terms of a 'normative model' each individual being considered to have a 'normal sleep pattern' and any deviation from this producing impairments in performance and/or alterations of mood. If 'normal' sleep patterns are held to consist of relatively fixed amounts of delta and REM sleep, then there appears to be little evidence supporting this assumption, since relatively large deviations from the 'norm' and even the selective deprivation of these two kinds of sleep appear to produce few effects on either performance or mood, although comparatively few studies have been conducted.

However, a number of studies have shown that there are diurnal variations in both task performance and mood and there is also evidence suggesting that the disturbance of sleep rhythms, whether those occurring within sleep, such as the REM cycle, or in particular, those which influence the location of sleep-wakefulness schedules within the circadian cycle, may produce considerable impairments both of performance and mood (Taub and Berger, 1969, 1973). Much of this research points to the importance for both performance and mood of maintaining sleep and wakefulness schedules within narrow temporal limits as well as, to a lesser extent, that of preserving the REM cycle. However, as yet, individual differences in the effect of sleep rhythm disturbances upon waking behaviour have been little explored.

\section{References}

Dement, W.C. \& Kleitman, N. (1957) Cyclic variations in EEG during sleep and their relation to eye movements, body motility and dreaming. Electroencephalography and Clinical Neurophysiology, 9, 673.

Feinberg, I. \& CARLSON, V.R. (1968) Sleep variables as a function of age in man. Archives of General Psychiatry, 18, 239.

Feinberg, I., KoresKo, R.L. \& Heller, N. (1967) EEG sleep patterns as a function of normal and pathological ageing in man. Journal of Psychiatric Research, 5, 107.

Hartmann, E. (1973) The Functions of Sleep. Yale University Press, New Haven, Connecticut.

HAURI, P. (1970) What is good sleep? In: Sleep and Dreaming (Ed. by E. Hartmann), International Psychiatry Clinics Series, Vol. 7. Little, Brown, Boston, Massachusetts.

Heron, A. (1956) A two part personality inventory for use as a research criterion. British Journal of Psychology, 47, 243.

JoHnSON, L.C. (1973) Are stages of sleep related to waking behaviour? American Scientist, 61, 326.

KAHN, E. \& Fisher, C. (1969) The sleep characteristics of the normal aged male. Journal of Nervous and Mental Disease, $148,477$.

Loomis, A.L., Harvey, E.N. \& Hobart, G. (1937) Cerebral states during sleep as studied by human brain potentials. Journal of Experimental Psychology, 21, 127.

MonRoE, L.J. (1967) Psychological and physiological differences between good and poor sleepers. Journal of Abnormal Psychology, 72, 255.

Rechtschaffen, A. \& KAlES, A. (Eds.) (1968) The Manual of Standardized Terminology, Techniques and Scoring System for Sleep Stages of Human Subjects. National Institutes of Health Publication No. 204. Washington D.C.

TAUB, J.M. \& BERGER, R.J. (1969) Extended sleep and performance: the Rip Van Winkle effect. Psychonomic Science, 16, 204.

TAub, J.M. \& Berger, R.J. (1973) Performance and mood following variations in the length and timing of sleep. Psychophysiology, 10, 559.

TunE, G.S. (1968) Sleep Patterns in the Human Adult (Unpublished report). Unit for Research on Occupational Aspects of Ageing, Department of Psychology, University of Liverpool.

TUNE, G.S. (1969) The influence of age and temperament on the adult human sleep-wakefulness pattern. British Journal of Psychology, 60, 431.

WEBB, W.B. (1965) Sleep characteristics of human subjects. Bulletin of the British Psychological Society, 18, 1.

Williams, R.L., KaRACAN, I. \& HURSCH, C.J. (1974) EEG of Human Sleep; Clinical Applications. Wiley, New York. 